\title{
Ações de Vigilância Sanitária na pandemia COVID-19
}

\section{Health Surveillance actions during the COVID-19 pandemic}

\section{Marcelo Pereira da Rochal* \\ Silvana Portella Lopes Cruz" (D)}

Alba Benemérita Alves Vilela"l' (ID

Vanda Palmarella Rodrigues"'I (iD)

\section{RESUMO}

Introdução: Em tempos de pandemia de COVID-19, a vigilância sanitária, assim como outros setores da saúde, precisou se adaptar para desenvolver suas atividades em um novo contexto desafiador. Objetivo: Descrever a atuação de profissionais da Vigilância Sanitária (Visa) no enfrentamento da pandemia da COVID-19. Método: Trata-se de um relato de experiência acerca do trabalho em Visa durante a pandemia em um Núcleo Regional de Saúde do estado da Bahia. Resultados: Foram relatadas ações realizadas em uma barreira sanitária, com acolhimento, palestra, testagem dos sintomáticos respiratórios e notificação, bem como a oferta de atividades de educação permanente em saúde, de forma presencial e on-line, por meio de reunião com os gestores municipais e demais ações realizadas pela equipe, tendo-se como destaque o caráter intrasetorial e intersetorial. Conclusões: A experiência do trabalho realizado coaduna com as propostas do Plano Estadual de Contingências para Enfrentamento do Novo Coronavírus. Salientamse as parcerias como de fundamental importância para os profissionais da Visa e de outros setores, frente à complexidade do momento pandêmico. A experiência ainda representa um aprendizado em lidar com novas ferramentas tecnológicas, que permitiram criar espaços de reflexão dos processos de trabalho, bem como de novas possibilidades de abordagem diante dos desafios atuais.

PALAVRAS-CHAVE: Vigilância Sanitária; COVID-19; Disseminação de Informação

\section{ABSTRACT}

Introduction: In times of the COVID-19 pandemic, health surveillance, as well as other health sectors, needed to adapt to develop its activities in a new challenging context. Objective: To describe the actions of health surveillance professionals in tackling the COVID-19 pandemic. Method: This is an experience report about the work in the sector of health surveillance during the pandemic in a Regional Health Center in the state of Bahia. Results: Actions undertaken in a health barrier were reported, including: welcoming, lectures, respiratory symptoms testing and notification, as well as the offer of continuing health education activities, in person and on-line, meetings with municipal managers, and other actions performed by the team, highlighting the intersectoral and intersectoral nature. Conclusions: The experience of the work conducted is consistent with the proposals of the Contingency Plan. Partnerships are of paramount importance for the professionals working in the sector of health surveillance and others, given the complexity of the pandemic moment. The experience also represents a learning process in dealing with new technological tools and allowed the creation of spaces for reflecting on work processes, as well as on new possibilities of approaching in light of the current challenges.

KEYWORDS: Health Surveillance; COVID-19; Information Dissemination 


\section{INTRODUÇÃOO}

No contexto atual de pandemia da COVID-19 (declarada em março de 2019) causada pelo novo coronavírus (SARS-CoV-2), que circula desde então em vários países e regiões do mundo ${ }^{1}$, muito se fala da vigilância sanitária, apesar de pouco se conhecer sua importância e atribuições no Sistema Único de Saúde (SUS).

A Lei Orgânica da Saúde define a vigilância sanitária como um conjunto de ações capazes de eliminar, diminuir ou prevenir riscos à saúde, bem como realizar intervenções nos problemas sanitários oriundos do meio ambiente, da produção e circulação de bens, além da prestação de serviços considerados de interesse da saúde ${ }^{2}$. Para tanto, os profissionais de saúde que atuam na Vigilância Sanitária (Visa) têm a prerrogativa do poder de polícia na área administrativa. Isso implica, muitas vezes, em limitar os direitos individuais dos cidadãos, pois busca-se a supremacia do interesse público.

O Brasil possui um Sistema Nacional de Vigilância Sanitária (SNVS) e os três entes federados: União, Estados e Municípios têm responsabilidades na execução das ações necessárias. Especificamente na Bahia, a coordenação das atividades do estado é de responsabilidade da Diretoria de Vigilância Sanitária, sediada em Salvador e de nove Núcleos Regionais de Saúde, instalados em macrorregiões. Nesses núcleos existem equipes de Visa com a função de prestar assessoria e apoio técnico aos municípios e realizar ações de forma complementar aos municípios, a exemplo de inspeções em estabelecimentos de alta complexidade ${ }^{3}$.

As ações de enfrentamento da pandemia na Bahia foram pautadas pelo Plano Estadual de Contingências para Enfrentamento do Novo Coronavírus ${ }^{4}$, instrumento orientador de gestores e instituições públicas de saúde na implantação e implementação de ações de proteção à saúde da população. Nesse plano estão previstas a realização de barreiras sanitárias nas rodovias interestaduais, o apoio institucional a municípios e estabelecimentos de saúde, a realização de atividades de educação permanente em saúde, dentre outras ações.

Em relação às barreiras sanitárias supracitadas, ainda se ressaltam os dispositivos legais que as autorizam, frente à emergência de saúde pública de interesse internacional, como a Lei Federal $\mathrm{n}^{\circ} 13.979$, de 6 de fevereiro de $2020^{5}$, no artigo $3^{\circ}$, inciso VI (cuja redação foi alterada pela Lei Federal $n^{\circ} 14.035$, de 11 de agosto de $2020^{6}$ ), na qual se prevê que autoridades poderão adotar medidas de restrição excepcional e temporária de rodovias, bem como a Resolução da Diretoria Colegiada (RDC) da Agência Nacional de Vigilância Sanitária (Anvisa) $n^{\circ} 353$, de 23 de março de $2020^{7}$, que delegou aos órgãos de vigilância sanitária estaduais a competência de "elaborar a recomendação técnica e fundamentada” para restrição de caráter excepcional e temporária em rodovias de locomoção interestadual e intermunicipal.

As barreiras sanitárias fazem sentido na medida em que há a necessidade de impedir a disseminação do vírus resultante da movimentação de pessoas contaminadas sintomáticas ou assintomáticas, com potencial de transmissão. Esta movimentação pode se dar por meio das tarefas cotidianas que permitem o contato entre as pessoas, no seu ambiente ou pelo deslocamento para outros municípios, estados ou mesmo de outros países ${ }^{8}$. As barreiras também propiciam a identificação e notificação de casos suspeitos de COVID-19, assim como também o retardo do avanço viral no território.

Vale ressaltar que o Plano Estadual de Contingência para o Enfrentamento do Novo Coronavírus do estado da Bahia ${ }^{4}$ contempla outras ações, além das barreiras sanitárias, que devem ser a elas associadas para enfrentamento da pandemia, como a estruturação da atenção primária à saúde (APS), ampliação do acesso do usuário à atenção hospitalar e estratégias de comunicação e cooperação nas esferas de gestão com vistas à organização do sistema de saúde.

Destarte, Machado et al. ${ }^{9}$ analisaram como a vigilância em saúde tem sido conduzida pela gestão nos estados do Nordeste do Brasil nesta pandemia e apontaram desafios sob os aspectos políticos gerenciais da vigilância em saúde e as articulações necessárias com os diversos setores que compõem a saúde, com vistas à intersetorialidade. Ainda ressaltam as ações integradas locorregionais como parte importante para o combate da pandemia.

Neste contexto pandêmico, a Visa apresenta um papel importante com atuação em várias linhas de frente, por meio de ações de esclarecimentos à população, educação em saúde aos profissionais e fiscalização dos estabelecimentos sujeitos à vigilância sanitária. Diante disso, este estudo propõe responder às seguintes questões norteadoras: como ocorreu a atuação da Visa regional em meio à pandemia? Como foi realizado o apoio aos municípios?

Nessa perspectiva, objetivou-se descrever a atuação de profissionais da Visa no enfrentamento da pandemia de COVID-19.

\section{MÉTODO}

Trata-se de um relato de experiência, de natureza qualitativa, a partir do trabalho desenvolvido em vigilância sanitária durante a pandemia de COVID-19 de um cirurgião-dentista e de uma enfermeira, ambos sanitaristas, do estado da Bahia.

O caráter qualitativo deste trabalho explica-se na exteriorização das impressões subjetivas de aprendizagem da experiência vivida pelos profissionais, pois, consoante Turato ${ }^{10}$, a metodologia qualitativa em saúde não estuda o fenômeno em si, mas procura apreender seu sentido para a vida das pessoas. Assim, as experiências vividas trazem diferentes significados que podem influenciar condutas a se tornarem valorosos exemplos para outros atores sociais ressignificarem as suas práticas.

O local do estudo é o Núcleo Regional de Saúde do Sudoeste do estado da Bahia, instância responsável pelo apoio e assessoria técnica aos municípios, bem como realização de ações de vigilância em caráter complementar e suplementar, cuja área de 
atuação abrange 74 municípios. 0 período da experiência relatada compreendeu o mês de março, quando começaram a ser notificados os primeiros casos suspeitos de COVID-19 na Bahia, ao mês de julho do ano de 2020.

As ações realizadas tiveram lastro nas orientações da Anvisa, da Diretoria de Vigilância Sanitária do Estado da Bahia (Divisa) e do Plano Estadual de Contingência para o Enfrentamento do Novo Coronavírus ${ }^{4}$, documento norteador para as equipes técnicas de diversas áreas, inclusive da vigilância em saúde.

Merece ressaltar que não se utilizou instrumento para a coleta de dados, pois o texto traz relatos narrados pelos autores, sem identificação de nomes, frases, dados ou qualquer outra informação dos participantes envolvidos (passageiros e motoristas em viagem, profissionais e gestores da saúde).

\section{RESULTADOS}

Atuar no combate à pandemia na área de vigilância sanitária apresenta muitos desafios face aos riscos de contaminação, bem como psicossociais e outros. Entretanto tem um lado positivo, de novos aprendizados e da possibilidade de contribuir com a prevenção de uma doença emergente e que merece tantos cuidados. Partindo disso, buscou-se compartilhar o trabalho realizado na barreira sanitária, no apoio aos municípios e no controle sanitário de estabelecimentos de saúde, incluindo-se aqueles com atendimento aos pacientes sintomáticos respiratórios e confirmados com a COVID-19.

\section{O trabalho na barreira sanitária}

No início da pandemia havia muitas incertezas e poucas respostas para tantas dúvidas, a exemplo da forma de transmissão da COVID-19 e das medidas de proteção à saúde. Para agravar a situação, a população brasileira estava exposta a especulações e notícias inverídicas, denominadas fake news. Até aquele momento ainda não havia muitos estudos publicados, de forma que as autoridades sanitárias e os profissionais de saúde se encarregavam de suprir as lacunas de conhecimento da sociedade, por meio de entrevistas nos mais variados meios de comunicação.

Diante de um ambiente tão desafiador, o Núcleo Regional de Saúde buscava construir um novo processo de trabalho em um ano totalmente atípico, visto que cada equipe já possuía suas ações de rotina programadas. Então, o planejamento elaborado para 2020 precisou ser revisto, na busca de enfrentamento da pandemia.

Uma das primeiras medidas constituiu-se no afastamento dos trabalhadores da saúde considerados como grupo de risco, como aqueles que tinham mais de 60 anos e estavam imunodeprimidos. Os demais trabalhadores ficaram em seus postos de trabalho ou foram para a sala de situação de saúde, composta por uma equipe de vigilância responsável por acompanhar a situação da pandemia regional, dar apoio às equipes de vigilância epidemiológica, divulgar boletins com informações epidemiológicas da COVID-19, dentre outras atividades importantes.
Por sua vez, os trabalhadores sem comorbidades foram atuar na linha de frente, em uma barreira sanitária instalada em importante rodovia interestadual, próximo da divisa com o estado de Minas Gerais. Para essa ação, foram alocados muitos trabalhadores da Visa e de outros setores, além de contar com o apoio da Polícia Militar e do Posto Fiscal, que permitiu o uso de suas instalações, como sala para refeições e sanitários. Esse posto é um local obrigatório de parada de veículos de transporte de mercadorias, como caminhões e carretas, para apresentação de notas fiscais dos produtos, portanto um local estratégico para o tipo de atividade proposta.

Pela primeira vez o núcleo estava implantando uma barreira sanitária, o que se constituía em um desafio para os profissionais de saúde não acostumados a desenvolver esta ação. Na barreira eram abordados os motoristas de transporte coletivo (vans e ônibus) e as pessoas que estavam em viagem, entre estes muitos motoristas que vinham das regiões Sul e Sudeste para entrega de mercadorias no Nordeste e muitos passageiros oriundos do Rio de Janeiro e São Paulo, por exemplo. Eram muitas histórias contadas. Grande parte das pessoas retornava ao Nordeste em decorrência do desemprego, desalentadas e sem perspectiva de retorno ao trabalho em curto espaço de tempo. Era uma situação triste, mas também de esperança por uma vacina. Muitos queriam saber quando ia terminar a pandemia ou quando se descobriria a cura da doença.

Os ônibus chegavam lotados, pois naquele momento o epicentro da doença era o Sudeste. Muitas pessoas chegavam sem máscara, cansadas da longa viagem, oportunidade em que eram acolhidas e convidadas a participar de uma palestra sobre prevenção da COVID-19. Além disso, era aferida a temperatura, realizada a notificação de suspeitos e a testagem de pessoas sintomáticas.

0 grande problema era que poucos tinham sintomas gripais e a impressão que se tinha era que o trabalho não tinha sentido. Ademais, vinham aglomerados, sentados próximos aos outros passageiros, em um ambiente com grande potencial de contaminação. No entanto, a ação educativa era bem proveitosa, pois se percebia participação proativa e ao final sempre tinha o agradecimento pelo trabalho realizado.

Nessa perspectiva, enquanto parte da equipe abordava os passageiros do transporte coletivo, outra parte realizava o mesmo trabalho com os caminhoneiros. Vale ressaltar que parte dessa categoria aceitou bem o trabalho realizado pela equipe da saúde. No entanto, parte deles questionava a falta de distribuição de luvas, máscaras e álcool em gel. Outros diziam que era perda de tempo, pois o vírus era invenção da China e que todos deveriam sair de casa para trabalhar e não somente alguns, pois a economia teria consequências e as pessoas poderiam passar fome. Mesmo assim, tentava-se dar uma explicação científica para os fatos, mesmo diante da dúvida quanto às evidências científicas até então produzidas.

Era um trabalho cansativo, pois se trabalhava em turnos das 7 às $19 \mathrm{~h}$, utilizando-se equipamentos de proteção individual (EPI), como máscara, touca e protetor facial em diversas condições 
climáticas, como em dias de muito calor, frio ou chuva, o que gerava desconforto. Mas também tinha um lado bom, como a integração, o apoio mútuo, os lanches e as alegrias compartiIhadas. À medida que a disseminação da COVID-19 crescia na Bahia, aumentava o descrédito quanto à efetividade da barreira sanitária, com sua interrupção no mês de maio.

\section{Apoio aos municípios vinculados ao Núcleo Regional de Saúde}

Após o fim da barreira sanitária, houve maior tempo para a dedicação às atividades direcionadas ao apoio dos municípios. Nesse aspecto, as ações de Visa de apoio institucional às equipes municipais foram realizadas majoritariamente por meio de uso de redes sociais, telefone, tendo em vista à substituição das atividades presenciais pelas atividades mediadas pela tecnologia no período da pandemia.

Nesse apoio se destacaram as atividades educativas com abordagem de conteúdos para atender as necessidades apontadas pelos coordenadores das Visa municipais, sendo a maioria desenvolvida de forma on-line.

Foi interessante desenvolver atividades com o apoio das novas tecnologias, o que permitiu oferecer momentos de educação permanente em saúde para os profissionais de saúde, de uma forma diferente das atividades presenciais. Os temas estavam relacionados aos assuntos pertinentes à pandemia, como ações de segurança do paciente e controle de infecção e a proteção da saúde do trabalhador no contexto da pandemia. As atividades tiveram a participação dos setores de Visa e saúde do trabalhador.

Outro aspecto importante decorreu da participação nas reuniões virtuais de pessoas de outras regiões da Bahia e até de outros estados, desde que tivesse acesso a um equipamento conectado à internet, como notebook ou celular. Mesmo à distância foi possível interagir por mensagem pelo chat ou por participação oral com o uso do microfone, o que colaborou com uma discussão qualificada dos assuntos tratados. Como ponto negativo, se destacou a queda de conexão pontual de alguns participantes que dependiam de um bom sinal de internet.

No entanto, houve também a oferta de ações de educação permanente em saúde de forma presencial, com abertura de espaço para discussão do processo de trabalho de ações que até então eram pouco discutidas, tais como a higienização das ambulâncias, manejo de resíduos de serviços de saúde e normas de segurança para os trabalhadores que realizavam a coleta dos resíduos urbanos. Foram momentos importantes, pois possibilitaram a integração do setor saúde com os diversos setores da gestão municipal, bem como a revisão das rotinas, dos fluxos de trabatho e a responsabilização das atribuições nas equipes.

Uma demanda interessante em relação ao apoio do núcleo aos municípios, ocorreu em razão de queixas de trabalhadores de algumas vigilâncias municipais sobre as condições de trabalho, como longas jornadas, não pagamento de direitos trabalhistas, baixa colaboração da população quanto ao distanciamento social e baixa capacidade da Visa em atender às muitas demandas da comunidade, muitas delas de não competência do setor. Isso motivou a vigilância regional a se posicionar com uma carta endereçada aos gestores municipais da saúde, para esclarecimentos sobre o papel da Visa, além de ressaltar a importância da melhoria das condições de trabalho e garantia de direitos trabalhistas.

Essa carta foi lida e discutida em reunião on-line com os Secretários de Saúde, no intuito de sensibilizá-los a dar o suporte necessário às equipes de Visa, inclusive do ponto de vista psicológico, dado o estresse vivenciado por aqueles que atuaram na linha de frente da pandemia.

Ações de controle do risco sanitário em estabelecimentos de saúde

Com o avanço da pandemia houve uma crescente contaminação de trabalhadores da saúde e de pacientes, inclusive com óbitos. Ao mesmo tempo, começaram a surgir denúncias a respeito da falta de EPI e das condições inadequadas de trabalho. Nesse sentido, foram realizadas inspeções sanitárias, mesmo diante do risco dessa ação.

Essas inspeções, realizadas em unidades de atendimento ao sintomático respiratório e aos pacientes adoecidos pela COVID-19, como hospitais, Unidades de Pronto Atendimento e Unidades de Atendimento ao Sintomático Respiratório, tinham como finalidade observar os aspectos preconizados nas normas sanitárias, como estrutura física e equipamentos adequados e higienização do local, além de identificar riscos relacionados ao trabalho e à proteção da saúde dos trabalhadores da saúde. Portanto, o enfoque foi educativo e propositivo, diferindo da face mais visível da vigilância sanitária, a fiscalizatória e punitiva.

É claro que as irregularidades foram apontadas em relatórios, mas procurou-se o apoio tanto dos trabalhadores, bem como da gestão para as mudanças necessárias. Desta forma, foram realizadas reuniões com os gestores e o assunto foi pautado nas agendas da Comissão Intergestores Regional (CIR), colegiado no qual participam os gestores municipais da saúde e representantes da Secretaria Estadual de Saúde.

Integração entre a Vigilância Sanitária e a vigilância epidemiológica

Frequentemente se fala da pouca integração entre a Visa e as demais vigilâncias. É notória a compartimentalização destes setores, cada qual no seu espaço e nas suas "caixinhas". Neste relato, mostra-se que é possível a realização de um trabalho articulado e em conjunto com outras áreas. Nesta perspectiva, o período de pandemia favoreceu um trabalho que envolveu a Visa, a vigilância epidemiológica, a atenção básica bem como outros setores do Núcleo Regional de Saúde, em atividades relacionadas aos Sistemas de Informação em Saúde (SIS) e produção de boletins informativos, por exemplo.

Diante da necessidade de apoio institucional aos municípios, realizou-se conjuntamente a revisão dos sistemas de notificação dos casos suspeitos e confirmados de COVID-19, efetivando a limpeza 
da base de dados municipais, com análise de cada caso notificado. Semanalmente era emitido um boletim para publicização da situação epidemiológica. Havia a necessidade de que estas informações refletissem a realidade, com o real número de casos confirmados, descartados e em monitoramento. Assim, a partir do rastreamento e da análise de cada caso, realizou-se a orientação das equipes municipais. A partir deste trabalho, procedeu-se à disponibilização dos dados oficiais à gestão, o que permitiu melhor conhecimento da situação da pandemia na região.

\section{DISCUSSÃO}

Atuar na linha de frente durante a pandemia de COVID-19 tornou-se um grande desafio para os profissionais de saúde, que lutam contra o novo coronavírus e precisam conviver com a gestão caótica da pandemia, dado o descompasso entre a atuação do governo federal, dos estados e dos municípios. A esse respeito se destacam contradições na adoção de medidas de controle da pandemia entre entes federados, visto que o presidente da República deu diversas declarações minimizando os impactos da COVID-19, contra as medidas de prevenção defendidas pela Organização Mundial da Saúde (OMS) e pelo próprio Ministério da Saúde, enquanto estava sob o comando do Ministro Henrique Mandetta até o mês de abril 2020. O Presidente ainda questionou ações de estados e municípios sobre as medidas de isolamento social, uso de máscara e de restrição das atividades econômicas consideradas não essenciais ${ }^{11}$.

Esse comportamento do Presidente e de outras autoridades federais refletiu na adesão de parte da população que entendia que a pandemia não era grave e que as medidas de prevenção recomendadas pela ciência não deviam ser seguidas. No diálogo com os caminhoneiros na barreira sanitária era perceptível essa divisão de opiniões entre os que estavam acolhendo bem o trabalho realizado na rodovia pela área de saúde e aqueles que negavam as recomendações da ciência. Era uma luta desigual, contra o vírus e contra o negacionismo da pandemia.

Em tempos de luta difícil contra um inimigo invisível, o vírus, e outros inimigos visíveis, como os que não acreditam na ciência, foi importante contar com parcerias, e foi o que fez a Visa, que compôs um trabalho integrado com outras áreas técnicas do núcleo e em articulação com os municípios da área de abrangência. Salienta-se que as ações realizadas estavam previstas no Plano de Contingências ${ }^{4}$ que visava enfrentar de forma rápida e coordenada a pandemia do novo coronavírus no estado da Bahia.

Esse plano preconiza as diretrizes para a organização dos serviços assistenciais, mas também foca nas ações de vigilância em saúde. O que se propõe é um trabalho articulado com a rede de atenção em saúde. Dessa forma, ao analisar a experiência aqui relatada, se percebe que ela coaduna com as orientações emanadas da Secretaria Estadual de Saúde a qual o núcleo está vinculado ${ }^{4}$.

O trabalho realizado, a exemplo da barreira sanitária, é uma ação atípica para a vigilância, visto que não são somente realizadas atividades educativas, mas também existe um viés de controle sobre as pessoas, com aferição da temperatura, imposição de medidas de isolamento e distanciamento social, o que limita as liberdades individuais. Como a barreira sanitária e outras ações não fazem parte da rotina da vigilância em saúde, entende-se a necessidade de melhor regulamentação, visto que novas emergências em saúde pública poderão ocorrer, face aos crescentes riscos vivenciados pela sociedade.

Ademais, a eficiência das barreiras sanitárias com o intuito de impedir parcialmente o fluxo das pessoas e de conter o avanço do coronavírus é questionada por Ferreira ${ }^{12}$. Ele afirmou que as barreiras se mostram insuficientes e, a cada dia, novos casos eram registrados em diversos locais. Segundo este autor, todas as barreiras, mesmo as mais rigorosas, possuem certa permeabilidade em que as pessoas atravessam e levam o vírus para outros lugares. Continua inferindo da necessidade de associar outras medidas, tais como o distanciamento social e proteção social para tentar aumentar a eficiência destas práticas. Contudo, ressaltou que, apesar das limitações apontadas, as barreiras sanitárias tiveram impacto positivo no atraso da incidência epidêmica e dando mais tempo para que os sistemas de saúde locais montassem estrutura para atender os casos da doença.

Nesta perspectiva, as ações de controle de uma pandemia devem ser iniciadas antes da identificação de casos suspeitos, por meio de medidas de contenção envolvendo o rastreio das pessoas originárias de regiões onde o vírus já foi detectado, no intuito de atrasar a transmissão comunitária. Após a detecção de casos confirmados passam-se para as ações que visem à transmissão do agravo para as pessoas mais vulneráveis e à adoção das medidas de isolamento social para os casos identificados, seguidas de medidas mais rigorosas que impeçam ou diminuam a movimentação de pessoas no meio social. Estas estratégias têm sido objeto de discussões entre os pesquisadores considerando suas consequências e impactos econômicos e sociais ${ }^{13}$.

Vale ressaltar que o trabalho aqui relatado esteve consorciado com atividades educativas para a população, não se limitando em apenas restringir o fluxo de pessoas, aferir a temperatura ou outros procedimentos. Palácio e Takenami ${ }^{14}$ acrescentaram os desafios para a realização da educação popular neste cenário pandêmico, apontaram a dificuldade dos profissionais de saúde da linha de frente para conseguirem adesão às recomendações das autoridades sanitárias nas ações de educação à população, e ainda afirmaram que um dos maiores desafios atuais é a educação em saúde.

Trabalho semelhante sobre ações de controle sanitário da pandemia da COVID-19 revela a importância das barreiras sanitárias, apontadas como estratégias que contribuem para a implementação de ações de vigilância, assim como uma oportunidade de ampliar acesso à informação por parte de viajantes ${ }^{8}$.

Quanto às atividades de educação permanente em saúde para os profissionais dos municípios, percebeu-se a importância da revisão dos fluxos de trabalho e práticas cotidianas que, de algum modo, estavam sendo negligenciadas nas rotinas. Ribeiro et al. ${ }^{15}$ acrescentaram que estes espaços nas suas experiências práticas permitiram uma reflexão crítica do processo de trabalho e que 
os próprios trabalhadores por meio da autoavaliação identificaram os desafios e sugeriram propostas alternativas na direção de resolução de problemas.

Diante de tantas dificuldades para o trabalho realizado na saúde, com milhares de mortes e descaso de parte da gestão pública, deve-se refletir sobre a comunicação com a sociedade, entendendo a importância de uma pedagogia libertadora capaz de implicar os sujeitos. Nesse sentido, cabe lembrar que Henrique e Vasconcelos ${ }^{10}$ disseram que, ao mesmo tempo que a população é vítima da doença ou de suas consequências, também é testemunha, observadora, analista, torcedora e protagonista, com responsabilidades relacionadas à prevenção, ao cuidado e à mitigação do sofrimento. Parece óbvio, mas é importante frisar que o enfrentamento da pandemia é responsabilidade não somente dos profissionais e das autoridades da saúde, mas de toda a população, sendo assim uma responsabilidade compartilhada com toda a sociedade.

\section{CONCLUSÕES}

Por meio desse relato foi possível descrever múltiplas ações desenvolvidas pela Visa regional em meio à pandemia, em atendimento ao que se preconiza no Plano Estadual de Contingências para o Enfrentamento do Novo Coronavírus do estado da Bahia.

Nesse ponto se destacaram algumas ações realizadas em parcerias intrasetoriais e intersetoriais, a exemplo da barreira sanitária. As ações conjuntas são de primordial importância, dada a complexidade de atuação frente à uma pandemia que tem ceifado tantas vidas e reforça a necessidade de "sair da caixinha”, pois é possível e se deve enfrentar problemas complexos de forma articulada com outras áreas.

Outro ponto importante foi o apoio à gestão municipal, especialmente por meio de ofertas de educação permanente, o que se constituiu um aprendizado para os profissionais de saúde em lidar com outras ferramentas de trabalho. Ainda oportunizou espaços de reflexão sobre os processos de trabatho e de novas possibilidades de encarar os desafios impostos neste período pandêmico.

Por fim, fica a expectativa de dias melhores para os brasileiros, a partir de um redirecionamento da gestão da pandemia e de maior apoio aos profissionais da Visa e de outros setores que batalham na linha de frente contra esse vírus mortal.

\section{REFERÊNCIAS}

1. Pan-American Health Organization - PAHO. Folha informativa: COVID-19 (doença causada pelo novo coronavírus). New York: Pan-American Health Organization; 2020[acesso 20 nov 2020]. Disponível em: https: / /www.paho.org/bra/ index.php?option=com_content\&view=article\&id=6101: covid19\&Itemid $=875$ \#historico

2. Brasil. Lei $\mathrm{N}^{\circ} 8.080$, de 19 de setembro de 1990 . Dispõe sobre as condições para a promoção, proteção e recuperação da saúde, a organização e o funcionamento dos serviços correspondentes e dá outras providências. Diário Oficial União. 20 set 1990.

3. Governo do Estado da Bahia. Resolução CIB Nº 249, 30 de dezembro de 2014. Aprova ad referendum a presente revisão da resolução CIB Nº 084/2011 nos termos constantes dos anexos I, II e III que apresentam os princípios gerais e estabelece as ações de competência do estado e dos municípios na organização, execução e gestão das ações do Sistema Estadual de Vigilância em Saúde do Estado da Bahia, de forma compartilhada, solidária, regionalizada e descentralizada. Diário Oficial Estado. 31 dez 2014.

4. Secretária da Saúde do Estado da Bahia - Sesab. Plano estadual de contingências para enfrentamento do novo coronavírus SARS CoV2. Salvador: Secretária da Saúde do Estado da Bahia; 2020[acesso 7 dez 2020]. Disponível em: http: / /www. saude.ba.gov.br/wp-content/uploads/2020/06/Plano-deContinge\%CC\%82ncia-Coronav\%C3\%ADrus-Bahia-2020-2606.pdf

5. Brasil. Lei $N^{\circ} 13.979$, de 6 de fevereiro de 2020. Dispõe sobre as medidas para enfrentamento da emergência de saúde pública de importância internacional decorrente do coronavírus responsável pelo surto de 2019. Diário Oficial União. 7 fev 2020.
6. Brasil. Lei $\mathrm{N}^{\circ} 14.035$, de 11 de agosto de 2020 . Altera a lei $N^{\circ} 13.979$, de 6 de fevereiro de 2020, para dispor sobre procedimentos para a aquisição ou contratação de bens, serviços e insumos destinados ao enfrentamento da emergência de saúde pública de importância internacional decorrente do coronavírus responsável pelo surto de 2019 . Diário Oficial União. 12 ago 2020.

7. Agência Nacional de Vigilância Sanitária - Anvisa. Resolução $\mathrm{RDC}^{\circ}$ 353, de 23 de março de 2020. Delega ao órgão de vigilância sanitária ou equivalente nos estados e no Distrito Federal a competência para elaborar a recomendação técnica e fundamentada relativamente ao estabelecimento de restrições excepcionais e temporárias por rodovias de locomoção interestadual e intermunicipal. Diário Oficial União. 23 mar 2020.

8. Martins EHC, Santos IP, Santos MS, Kretlli PGNC, Marques SHP, Cordeiro TMO. Operação especial de barreiras sanitárias para enfrentamento do vírus Sars-CoV-2, Bahia, 2020. Rev Baiana Saúde Pública. 2021;45(esp.2):92-107. https://doi.org/10.22278/2318-2660.2021.v45.nEspecial_2.a3484

9. Machado MF, Quirino TRL, Souza CDF. Vigilância em saúde em tempos de pandemia: análise dos planos de contingência dos estados do nordeste. Vigil Sanit Debate. 2020;8(3):70-7. https://doi.org/10.22239/2317-269x.01626

10. Turato ER. Métodos qualitativos e quantitativos na área da saúde: definições, diferenças e seus objetos de pesquisa. Rev Saúde Pública. 2005;39(3):1-8. https://doi.org/10.1590/\$0034-89102005000300025

11. Henriques CMP, Vasconcelos W. Crises dentro da crise: respostas, incertezas e desencontros no combate à pandemia da COVID-19 no Brasil. Estud Av. 2020;34(99):120. https://doi.org/10.1590/s0103-4014.2020.3499.003 
12. Ferreira S. Sobre a eficiência das barreiras sanitárias restritivas para conter o progresso do COVID-19: uma modelagem matemática simples. Scielo Preprints. 2020:1-13 Disponível em: https://doi.org/10.1590/SciELOPreprints.691

13. Werneck GL, Carvalho MS. A pandemia de COVID-19 no Brasil: crônica de uma crise sanitária anunciada. Cad Saúde Pública. 2020;36(5):1-4. https://doi.org/10.1590/0102-311X00068820
14. Palácio MAV, Takenami. COVID-19: o desafio para a educação em saúde. Vigil Sanit Debate. 2020;8(2):10-5. https://doi.org/10.22239/2317-269x.01530

15. Ribeiro MC, Bedoya SO, Ferreira VA, Oliveira AMC. Desafios e oportunidades de repensar o processo de trabalho em vigilância sanitária: um relato de experiência. Vigil Sanit Debate. 2020;8(4):1-5. https://doi.org/10.22239/2317-269x.01516

\section{Contribuição dos Autores}

Rocha MP, Cruz SPL, Rodrigues VP - Concepção, planejamento (desenho do estudo) e redação do trabalho. Vilela ABA - Redação do trabalho. Todos os autores aprovaram a versão final do trabalho.

Conflito de Interesse

Os autores informam não haver qualquer potencial conflito de interesse com pares e instituições, políticos ou financeiros deste estudo.

Licença CC BY-NC atribuição não comercial. Com essa licença é permitido acessar, baixar (download), copiar, imprimir, compartilhar, reutilizar e distribuir os artigos, desde que para uso não comercial e com a citação da fonte, conferindo os devidos créditos de autoria e menção à Visa em Debate. Nesses casos, nenhuma permissão é necessária por parte dos autores ou dos editores. 